\title{
Headache in an Italian pediatric emergency department
}

\author{
Paola Scagni · Rosaura Pagliero
}

Received: 2 October 2007/Revised: 3 January 2008/Accepted: 8 January 2008/Published online: 5 February 2008

(C) Springer-Verlag 2008

\begin{abstract}
The objective of this study was to assess epidemiology, diagnostic work-up, treatment and follow-up of children presenting to emergency department (ED) with headache. Records of visits for non-traumatic headache to the ED of a pediatric hospital over a period of 12 months were retrospectively reviewed. Headache center charts were analyzed one year after. Five-hundred and fifty patients (1\% of all ED visits) were included. Spectrum of diagnoses was: primary headache $(56.7 \%)$, with $9.6 \%$ of migraine; secondary headache $(42 \%)$; unclassified headache (1.3\%). Viral illnesses accounted for $90.5 \%$ of secondary headaches. A serious disorder was found in $4 \%$ of patients. Forty-four patients $(8 \%)$ underwent neuroimaging studies, with $25 \%$ of abnormal findings. Only 223 patients $(40.5 \%)$ received pharmacological treatment. On discharge, 212 patients $(38.5 \%)$ were referred to headache center and 114 (20.7\% of all patients) attended it. ED diagnosis was confirmed in $74.6 \%$ of cases. Most of ED repeated visits $(82.6 \%)$ occurred in patients not referred to headache center at discharge from first ED visit. The most frequent diagnosis was primary headache; viral illnesses represented the majority of secondary headaches. Underlying serious disorders were associated with neurological signs, limiting the need of diagnostic investigations. Well structured prospective studies are needed to evaluate appropriate diagnostic tools, as well as correct therapeutic approach of pediatric headache in emergency. Collaboration with headache center might limit repeated visits and provide a correct diagnostic definition.
\end{abstract}

P. Scagni $(\bowtie) \cdot$ R. Pagliero

Department of Pediatrics, Headache Center, Children's Hospital

Regina Margherita, P.zza Polonia 94, 10126 Torino, Italy

e-mail: scagni@hotmail.com
Keywords Headache - Migraine - Emergency department $\cdot$ Childhood

\section{Introduction}

Headache is an extremely common condition in childhood, with a prevalence of $40-50 \%$ by age 7 and $70-75 \%$ by age 15 [1]. The majority of headaches are benign and selflimited and can be accurately diagnosed on the basis of a careful history and physical examination; although the frequency of headaches resulting from serious organic causes is low, this population may include children with conditions which require immediate medical care. While most studies are based on adults [2-9], few surveys have examined the chief complaint of headache and its epidemiological impact in the pediatric emergency department (ED) [10-13].

The purpose of this study was to: determine the demographic and clinical characteristics of patients presenting to the pediatric ED with a complaint of headache; investigate the diagnostic work-up and therapeutic approach employed in these children; study the impact of a specific follow-up.

\section{Subjects and methods}

A retrospective study was performed in the ED of Regina Margherita Children's Hospital, a pediatric hospital which serves as the primary referral center for all pediatric diseases in the geographic area. All patients with a chief complaint of headache attending the ED between 1 October 2003 and 30 September 2004, were eligible for inclusion. A search of the ED database was carried out to identify all patients eligible. Patients were excluded from 
the study if any of the following were present: posttraumatic headache, bacterial meningitis, notes not available for review or documentation not sufficient. During the period studied, 53.794 visits were made to the ED; of these, database search identified 550 patients whose main complaint was headache. Seven records had been excluded because of insufficient data. For each included patient a standardized data collection form was completed, recording the following data: patient age and sex, history of headache and associated symptoms, physical examination, results of laboratory exams, neuroimaging investigations and consultations, therapies administered and final diagnosis. Final diagnosis was considered to be that recorded by the ED doctor for patients discharged and that recorded on the discharge summary for those admitted. Headaches were divided into primary and secondary. Headaches were defined as secondary if a specific cause was identified, and they were further divided into neurological and secondary to viral illnesses or respiratory infections. "Serious/pathological" diagnoses were defined as any disorder requiring a prompt intervention in ED, included any organic and structural involvement of the central nervous system; for the purposes of this study, migraine was not considered a pathological disorder. After discharge from the ED and according to ED doctor final decision, many children with a diagnosis of primary headache were referred to our specialist headache center in order to be part of a follow-up program. Twelve months after ED referring, headache center records of each patient were reviewed.

\section{Results}

During the period studied, 526 patients made 550 visits to the ED for headache complaints: $503(95.6 \%)$ children made a single visit, $22(4.2 \%)$ made two visits and one made three visits $(0.2 \%)$. Overall, non-traumatic headache accounted for $1 \%$ of total ED visits during the year studied. There were 265 males and 261 females, with a mean age of 8.8 years. The age ranges were as follows: $86(15.6 \%)$ preschool children (0-5 years), $250(45.5 \%)$ primary schoolage children (6-10 years) and $214(38.9 \%)$ adolescents (11-16 years); five patients were aged 2 years or younger. Number of repeated visits was $23(4.2 \%)$. In $56.5 \%$ of cases (13 out of 23) return visit occurred in the week following the first ED visit (mean time 3 days) and was due to headache unresponsive to previous ED therapy; in remaining ten cases $(46.5 \%)$ return visit was related to a new episode of headache, with a mean time of 84 days after the first ED visit. Only four of these 23 patients $(17.4 \%)$ had been referred to headache center at discharge from first ED visit.
Ninety-seven patients were admitted to hospital for further investigations, with an admission rate of $17.6 \%$. Reasons for admission included: abnormal findings on history or physical examination (such as long lasting headache, personality changes, vertigo, visual, motor or sensory symptoms, lethargy or loss of consciousness) in 55 patients (56.7\%); severe vomiting in 25 patients (25.8\%); fever of unknown origin in nine cases $(9.3 \%)$; associated diseases, including ventriculoperitoneal (VP) shunt, brain malformations, cardiopathy and previous cerebrovascular diseases in eight children (8.2\%). In six cases, hospital admission followed a repeated visit to the ED. Seven patients refused admittance.

Data regarding final diagnosis were as follows: 312 cases $(56.7 \%)$ were diagnosed as having a primary headache disorder, 231 cases (42\%) had a secondary headache and seven $(1.3 \%)$ were discharged with a diagnosis that could not clearly account for their headache (such as syncope or abdominal pain). Migraine accounted for $5.4 \%$ of all visits (30 of 550) and for $9.6 \%$ of primary headaches; among patients with migraine, in 19 cases $(63.3 \%)$ final diagnosis was made only after hospital admission. Acute viral and respiratory illness (id upper respiratory infection, pharyngitis, sinusitis and gastritis) represented the most frequent cause of secondary headache $(90.5 \%)$ and $38 \%$ of all attending headaches (209 of 550). Other less common causes of secondary headaches included hypertension, carbon monoxide exposure and neurological disorders. A "pathological" diagnosis was made in 22 patients, with an overall frequency of $4 \%$. Neurological disorders were found in $3 \%$ of all visits (17 cases), including two cases of VP shunt malfunctions, five cases of seizures, two brain tumors, two aseptic meningitis, three cerebrovascular diseases (one intracranial hemorrhage, one ischaemic attack and one venous sinus thrombosis) and three vestibular neuritis; remaining "pathological" diagnoses included four cases of intoxication and one case of hypertension. All patients with neurological underlying diseases had a $100 \%$ rate of any pathological findings on history or physical examination, such as loss of consciousness, lethargy, vomiting, vertigo, hemiparesis, papilledema and focal motor signs. All patients with "pathological" disorders were appropriately diagnosed and treated on their first visit to the ED. Table 1 summarizes headache etiologies of these patients.

A total of 155 specialist consulting visits were required; in particular, neurological and ophthalmologist visits accounted respectively for $22.6 \%$ (35 of 155 ) and $44.5 \%$ (69 of 155) of these consultations. Retrospectively it was not possible to appreciate reason of consulting visits from ED database. Electroencephalogram (EEG) was performed in 16 patients $(2.9 \%)$. Patients who underwent EEG presented on history or physical examination: focal motor or 
Table 1 Headache etiology in pediatric emergency department

\begin{tabular}{lcc}
\hline Diagnoses & $N=550$ & Percentage \\
\hline Primary headache & 312 & 56.7 \\
Not specified & 282 & 90.4 \\
Migraine & 30 & 9.6 \\
Secondary headache & 231 & 42 \\
Viral and respiratory infections & 209 & 90.5 \\
Neurological & 17 & 7.3 \\
Others $^{\text {a }}$ & 5 & 2.2 \\
Unclassified & 7 & 1.3 \\
\hline
\end{tabular}

a Others: hypertension and carbon monoxide intoxication

sensory signs (seven cases), vertigo (five cases), vomiting (two cases) or headache as the only symptom (two cases). Specific EEG abnormalities were observed exclusively on patients with signs suggestive of seizures. Forty-four patients $(8 \%)$ underwent structural brain imaging during hospital attendance; $6.8 \%$ of these were performed while the patient was under the care of the ED. Overall, 11 neuroimagings (25\%) detected an abnormal finding, including hydrocephalus in VP shunt, brain tumors and cerebrovascular diseases. In 10 out of 44 patients (22.7\%) structural brain imaging was performed because of nonspecific abnormalities observed at EEG; in all cases a normal radiological finding was observed. The use of neuroimaging revealed in three cases the discovery of incidental benign abnormalities (Chiari I malformation, arachnoid cyst, non-specific white matter abnormalities).

Two-hundred and twenty three (40.5\%) patients received pharmacological treatment. Table 2 summarizes the medication profiles of these patients. Drug regimen and response to therapy was not clearly detailed on ED database records.

On discharge from ED, only 212 out of 312 patients $(67.9 \%)$ with primary headache were referred to our headache center; 114 of them (20.7\% of the total population) attended the center. Among these 114 children, headache center staff diagnosed migraine in 29 patients discharged from ED with a diagnosis of "primary headache not otherwise specified"; on the contrary, ED diagnosis of migraine was confirmed in all patients.

Table 2 Treatment of headache in pediatric emergency department

\begin{tabular}{lcc}
\hline Medication & $N=223$ & Percentage \\
\hline Acetaminophene & 160 & 71.7 \\
NSAIDs & 26 & 11.7 \\
Noramidopyrine & 16 & 7.2 \\
Opiates & 15 & 6.7 \\
Antiemetics & 6 & 2.7 \\
\hline
\end{tabular}

Only 2 out of 550 visits $(0.4 \%)$ to the ED for headache occurred in patients regularly followed in our headache center. In both cases reason for ED visit was headache associated with symptoms never experienced in the past.

\section{Discussion}

Although headache is a common complaint in childhood, [1] it is an unusual presenting symptom in ED. In our study, which represents the largest analysis of this complaint in a pediatric ED, [10-13] we found that headache accounts for $1 \%$ of all visits. Similar rates were reported in smaller pediatric series $(0.7-1.3 \%)$ [10, 13] and in adult studies (1-4\%) [2-4]. Regarding the Italian experience, data from adult analysis show a prevalence ranging from $0.6 \%$ for primary headaches in ED [5] to $0.8-2.35 \%$ of all ED complaints [6-9]; no Italian pediatric series exist. Compared to previously reported pediatric data, a similar high frequency in school-age children and a more homogenous male-to-female ratio was observed [11, 13].

Differential diagnosis of the patient presenting with headache is essential to rule out organic diseases and situation of immediate danger. Earlier studies have shown that the most common cause of headache in childhood is secondary headache, mainly in the setting of an upper respiratory infection [10-13]. Compared with previous pediatric reports, an unexpected data obtained in our population was the higher prevalence of primary headache diagnosis versus secondary forms, being the proportion of patients classified with primary headache $56.7 \%$ in our study versus $10 \%$ in Kan's [13] and 20\% in Burton's [10]. Similar proportion of primary headaches are usually reported in adults [9, 14] and suggest an increasing awareness of primary headache as clinical disorder in childhood. As regards secondary headaches, viral illness represented the most common diagnosis. The overall frequency of "pathological" diagnosis was low (4\%) and only a minority of children had neurological conditions such as proven VP shunt malfunction, brain tumor or cerebrovascular diseases. In all these patients, a detailed history and a careful neurological examination proved to be effective, enabling an appropriate diagnosis on first visit to ED. Taking into consideration that our hospital is the referring regional center for all pediatric diseases, subsequent "pathological" diagnoses in other hospitals are improbable.

Despite the high frequency of primary headache diagnosis, most of these patients were discharged with a diagnosis of "primary headache not otherwise specified" and only $9.6 \%$ of them were classified as having a migraine. Data observed may be the consequence of the methodological approach, which was retrospective. However, according 
Table 3 Neuroimaging studies performed in the evaluation of headache in pediatric emergency department: comparison to the literature

\begin{tabular}{lrll}
\hline Author & $n$ & $\begin{array}{l}n / \text { percentage } \\
\text { of neuroimaging }\end{array}$ & $\begin{array}{l}\text { Percentage of } \\
\text { abnormal findings }\end{array}$ \\
\hline Current study & 550 & $44(8)$ & 25 \\
Kan et al. ${ }^{[13]}$ & 130 & $53(41)$ & 10 \\
Burton et al. ${ }^{[10]}$ & 288 & $21(7.3)$ & 19 \\
Goldstein et al. $^{[3]}$ & 5198 & $490(14.5)$ & 5.5 \\
\hline
\end{tabular}

with previous published data [2, 5,7], the main impression is that most of ED pediatricians stop the diagnosis process upon the differential diagnosis between primary and secondary headache. A low experience in headache classification according to International Classification of Headache Disorders (ICHD) 2004 criteria can be supposed [15]. This might explain also the higher admission rate observed in our population (17.6\%) compared to previous studies [16]. According with adults series, admission was more common for migraine patients [7, 17].

With regard to diagnostic work-up, the number of specialist consulting visits requested by ED physicians was high (28.2\% of all patients), similar to frequencies reported by other studies (10-40\%) [8, 18]. Neuroimaging (head CT scan or MRI) was performed on $8 \%$ of children, which on first glance appeared high given that $96 \%$ of all patients were ultimately diagnosed with a benign disease. However, $25 \%$ of those who underwent neuroimaging were ultimately diagnosed with a "pathological" process. Despite suggestions that imaging could be overused, our rates are not excessive and are fairly lower than that reported in other studies performed in the ED setting (Table 3) [3, 10, 13]. Furthermore, $30 \%$ of radiological procedures with normal findings were performed because of non-specific abnormalities observed at EEG; moreover, all EEG performed in absence of seizures resulted normal or showed non-specific abnormalities. According to practice parameters published by the American Academy of Neurology and the Child Neurology Society [19], our data confirm that EEG is not recommended in the routine evaluation of headache, as it is unlikely to define an etiology and may lead to the discovery of incidental benign abnormalities, with subsequent unnecessary investigations and hospital admission. In general, diagnostic studies are seldom required in evaluating headache in children without clinical evidence of underlying structural lesions. Neuroimaging should therefore be limited to children presenting to the ED with headache associated with symptoms and neurological signs predictive for intracranial pathology and in those with underlying risk factors (such as VP shunt).

With regard to therapeutic approach, only a minority of patients $(40.5 \%)$ was treated with any type of analgesic.
The use of acetaminophen was prevalent, with more than $70 \%$ of patients treated this way; in a smaller proportion of our population other medications such as antiemetics and opioids were used. Interestingly, response to therapy was not clearly indicated in all ED records. Furthermore, all repeated visits occurring shortly after the first ED admission were due to headache unresponsive to previous ED therapy. It is unclear why pain relief is not a priority in pediatric ED, but it is possible that the severity of headaches in children presenting to ED is not as serious as that experienced by adults. The absence of clinical guidelines specifically designed for headache therapy in pediatric emergency may interfere also on this habit and on the choice of medications [13].

No previous pediatric study has investigated the usefulness of a specific follow-up program for these patients. Headache centers are dedicated to the treatment of primary headaches; they are uncommon in pediatrics and generally have a long waiting list. In our hospital there is a collaboration with the ED: according to ED doctors final decision, patients discharged with a diagnosis of primary headache or "headache not otherwise specified" are referred to our headache center and visited within $10-15$ days. We feel this alliance is very important and suggest it might play a role in containing the "repeaters" phenomenon showed by Maizels [20]. This is a very expensive cluster of patients, accessing the ED more than three times in a period of 6 months. In the US experience these are $10 \%$ of the total population, accounting for about $50 \%$ of headache-related visits to the ED; using the same parameters, we found a low percentage of repeaters in our population $(<1 \%)$. In particular, most of repeated visits $(82.6 \%)$ occurred in patients who hadn't been referred to headache center after first discharge from ED. Interestingly, only two patients regularly followed in headache center made a visit to the ED for headache complaints during the period studied. Neverthless, a prospective case-control study should be conducted to better define and assess the real impact of headache center on the "repeaters" phenomenon. Moreover, we suppose that collaboration between headache center specialists and ED staff might avoid unnecessary investigations in ED, as confirmed by the low rate of neuroimagings performed in our population compared with previous studies $[3,10,13]$. Last but not least, we consider the importance of providing a follow-up visit for a correct diagnostic definition according to ICHD-2004 criteria, as suggested by final diagnosis of migraine in children discharged from ED with a diagnosis of "primary headache not otherwise specified". Nevertheless, we found that only $67.9 \%$ of primary headaches presenting to ED were referred to headache center; we observed also a low family adherence (53.8\%) to headache center referral. It is possible that main concern of 
families attending ED is to receive reassurance that their children's headache is not caused by a life-threatening disease; similarly, the main purpose of ED pediatricians seems to be the exclusion of secondary "pathological" headaches. An exact diagnostic definition and a follow-up program are probably less significant for families and ED staff too.

In conclusion, headache is a symptom accounting for $1 \%$ of all visits in our pediatric ED. The high frequency in the general pediatric population and vagueness of symptoms make headache a worrying symptom with the need to exclude a situation of immediate danger. At the same time, the majority of headaches are benign or secondary to selflimited viral illnesses; serious conditions, such as neurological disorders, are rare and could be diagnosed on the basis of a careful history and a detailed neurological examination. Use of diagnostic investigations, which represent a biological and economical burden for the whole population, should therefore be limited. Pain relief doesn't seem a priority in pediatric ED; specifically designed guidelines are therefore needed in order to improve treatment of pediatric headache in the emergency setting. Collaboration with headache center staff, might limit repeated visits in ED, avoid unnecessary investigations, provide a correct diagnostic definition according to ICHD2004 criteria and ensure an appropriate follow-up.

\section{References}

1. Brna PM, Dooley JM (2006) Headaches in the pediatric population. Semin Pediatr Neurol 13(4):22-230

2. Morgenstern LB, Huber JC, Luna-Gonzales H et al (2001) Headache in the emergency department. Headache 41:537-541

3. Goldstein JN, Camargo CA, Pelletier AJ, Edlow JA (2006) Headaches in United States emergency departments: demographics, work-up and frequencies of pathological diagnoses. Cephalalgia 26:684-690

4. Ward TN, Levin M, Philips JM (2001) Evaluation and management of headache in the emergency department. Med Clin North Am 85:971-984
5. De Carli GF, Fabbri L, Cavazzuti L et al (1998) The epidemiology of migraine: a retrospective study in Italian emergency departments. Headache 38(9):697-704

6. Bono G, Antonacci F, Mancioli A et al (2006) The management of headache in the emergency department: critical issues. Neurol Sci 27:S59-S61

7. Cerbo R, Villani V, Bruti G et al (2005) Primary headache in emergency department: prevalence, clinical features and therapeutical approach. J Headache Pain 6:287-289

8. Relja G, Granato A, Capozzoli F et al (2005) Nontraumatic headache in the emergency department: a survey in the province of Trieste. J Headache Pain 6:298-300

9. Querzani P (2004) Guidelines for the management of headache in the emergency department. Neurol Sci 25:S190-191

10. Burton LJ, Quinn B, Pratt-Cheney JL, Pourani M (1997) Headache etiology in a pediatric emergency department. Pediatr Emerg Care 13(1):1-4

11. Lewis DW, Qureshi F (2000) Acute headache in children and adolescents presenting to the emergency department. Headache 40:200-203

12. Lewis DW (2001) Headache in the pediatric emergency department. Semin Pediatr Neurol 8(1):46-51

13. Kan L, Nagelberg J, Maytal J (2000) Headaches in a pediatric emergency department: etiology, imaging and treatment. Headache 40:25-29

14. Letch MJ (1980) Non-traumatic headache in the emergency department. Ann Emerg Med 4:404-409

15. Headache Classification Committee of the International Headache Society (2004) The international classification of headache disorders. Cephalalgia 24(suppl 1):1-151

16. Gahir KK, Larner AJ (2006) Primary headache disorder in the emergency department: perspective from a general neurology outpatient clinic. Emerg Med J 23:135-136

17. Blumenthal HJ, Weisz MA, Kelly KM et al (2003) Treatment of primary headache in the emergency department. Headache 43:1026-1031

18. Silberstein SD (1992) Evaluation and emergency treatment of headache. Headache 32:396-407

19. Lewis DW, Ashwal S, Dahl G et al (2002) Practice parameter: evaluation of children and adolescents with recurrent headaches: Report of the Quality Standards Subcommittee of the American Academy of Neurology and the Practice Committee of the Child Neurology Society. Neurology 59:490-498

20. Maizels M (2002) Health resource utilization of the emergency department headache "repeater". Headache 42:747-753 